J. Lake Sci. (湖泊科学) , 2011, 23(6): 919-925

http: //www. jlakes. org. E-mail : jlakes@niglas.ac.cn

(c) 2011 by Journal of Lake Sciences

\title{
蓝藻碎屑分解速率及氮磷释放形态的实验分析 *
}

\author{
李 柯 $^{1}$, 关保华 ${ }^{2 * *}$, 刘正文 ${ }^{2}$ \\ $(1:$ 华中农业大学水产学院,武汉 430070) \\ (2: 中国科学院南京地理与湖泊研究所湖泊与环境国家重点实验室, 南京 210008)
}

\begin{abstract}
摘 要: 在试验条件下研究了水华蓝藻堆积使叶绿素浓度达到 $1600 \mu \mathrm{g} / \mathrm{L}$ 时, 水体所含蓝藻形成的碎屑在水中的分解速 率及其营养盐释放情况. 结果表明: 蓝藻碎屑的总氮含量为 $88.56 \pm 4.10 \mathrm{mg} / \mathrm{g}$, 其在水体中有较高的分解速率, $2 \mathrm{~d}$ 内即分 解掉 $41.9 \%$ 的生物量; 蓝藻碎屑磷释放速率要高于氮, 但其导致的水体中溶解性总氮浓度的升高则较溶解性总磷持续时 间更长; 实验前 $4 \mathrm{~d}$, 溶解性无机氮 (DIN) 和有机氮 (DON) 浓度均有所升高, 之后 DIN 趋于稳定, 而 DON 则持续升高; DIN 中以铵态氮为主, 硝态氮、亚硝态氮含量较低; DON 中尿素氮比例仅为 $3 \%$, 说明其不是蓝藻分解释放的主要有机氮形式; 溶解性无机磷 (DIP)、溶解性有机磷 (DOP) 浓度在前 $4 \mathrm{~d}$ 均有所升高, 之后 DOP 逐渐转化为 DIP 并趋于稳定. 本实验结果 将有助于深人了解蓝藻水华对湖泊氮、磷循环的影响.
\end{abstract}

关键词: 蓝藻碎屑; 分解; 营养盐释放;氮形态; 磷形态

\section{Experiments on decomposition rate and release forms of nitrogen and phosphorus from the decomposing cyanobacterial detritus}

LI Ke ${ }^{1}$, GUAN Baohua ${ }^{2} \&$ LIU Zhengwen ${ }^{2}$

(1: College of Fisheries, Huazhong Agricultural University, Wuhan 430070, P. R. China)

(2: State Key Laboratory of Lake Science and Environment, Nanjing Institute of Geography and Limnology, Chinese Academy of Sciences, Nanjing 210008, P. R. China)

\begin{abstract}
The decomposition rate of cyanobacterial detritus and the release forms of nitrogen and phosphorus from the decomposing cyanobacterial detritus were investigated experimentally in this paper. Results showed that the content of total nitrogen of cyanobacterial detritus was $88.56 \pm 4.10 \mathrm{mg} / \mathrm{g}$; the decomposition rate of cyanobacterial detritus was high and there was $41.9 \%$ dry mass lost in two days. The release rate of total phosphorus of cyanobacterial detritus was faster than total nitrogen, but the increase in concentration of total dissolved nitrogen (TDN) in the water column lasted longer than total dissolved phosphorus (TDP) during the decomposing of cyanobacteria. After the increase in the first four days, the concentration of dissolved inorganic nitrogen (DIN) was kept constant but the concentration of dissolved organic nitrogen (DON) continued to increase. The ammonia was the main form of DIN in the water column, while the concentration of nitrate and nitrite was low during the decomposition. The Urea-N was not the main form of DON released by cyanobacterial detritus because the average percentage of Urea-N was low ( $3 \%$ of DON). The concentration of dissolved inorganic phosphorus (DIP) and dissolved organic phosphorus (DOP) increased in the first four days, and then DOP was transformed into DIP progressively. The present study demonstrated that the decomposition rate of cyanobacterial detritus is high, with releasing of organic and inorganic nitrogen and phosphorus. The result of present study shed light on understanding of the impact of cyanobacterial blooms on nitrogen and phosphorus cycling of lakes.
\end{abstract}

Keywords: Cyanobacterial detritus; decomposition; nutrient release; forms of nitrogen; forms of phosphorus

* 国家科技支撑计划项目 (2007BAC26B02)、国家“973”计划项目 (2008CB418104)、国家水体污染控制与治理科技 重大专项项目 (2009ZX07210-001-004) 和国家自然科学基金项目 (31070419) 联合资助. 2010 - 11-29 收稿; 2011-04-20 收修改稿. 李柯, 男,1986 年生, 硕士研究生;E-mail : lake918lake@ 163. com.

** 通讯作者;E-mail: bhguan@ niglas. ac. cn. 
蓝藻水华的大面积暴发是目前全世界面临的重大环境问题之一 ${ }^{[1]}$. 在富营养湖泊中, 一旦水文条件适 合, 蓝藻会大规模暴发, 堆积的蓝藻水华很快开始死亡, 形成大量有机碎屑 ${ }^{[2-3]}$, 同时开始腐烂分解, 并释放 大量的可溶性物质,包括氮、磷和有机碳等 ${ }^{[4-5]}$, 这些物质将显著改变水体中的营养盐含量及组成形态 ${ }^{[6]}$, 并 为水中浮游植物、附着生物、大型植物和细菌等提供大量营养. 由于生物对不同形态营养盐的吸收有差 别 ${ }^{[7]}$, 因此, 详细探究蓝藻水华分解释放的营养盐形态, 将有助于深人了解蓝藻暴发对水体营养盐循环的影 响程度及其机理. 太湖的富营养化和蓝藻水华问题已经持续了 20 多年, 且近年来有逐渐加重的趋势 ${ }^{[8]}$, 高 温季节暴发的蓝藻水华在东南风作用下向北部梅梁湾沿岸堆积, 使局部沿岸地区叶绿素浓度达到 $2000-$ $3000 \mu \mathrm{g} / \mathrm{L}^{[9]}$. 本实验旨在研究大量堆积的蓝藻碎屑颗粒在水体中的分解速率及其分解对水体不同形态的 氮磷营养盐浓度的影响, 从而深人了解蓝藻碎屑腐烂分解时的营养盐释放规律, 并为进一步研究蓝藻水华 对湖泊营养盐循环的影响奠定基础.

\section{1 材料与方法}

\section{1 实验材料}

表 1 实验用水初始水化学指标

Tab. 1 The nutrient conditions of initial water

\begin{tabular}{ll}
\hline 指标 & 浓度 $(\mathrm{mg} / \mathrm{L})$ \\
\hline 总氮 $(\mathrm{TN})$ & $2.603 \pm 0.049$ \\
溶解性无机氮 $(\mathrm{DIP})$ & $2.264 \pm 0.029$ \\
铵态氮 $\left(\mathrm{NH}_{4}^{+}-\mathrm{N}\right)$ & $0.230 \pm 0.021$ \\
硝态氮 $\left(\mathrm{NO}_{3}^{-}-\mathrm{N}\right)$ & $1.995 \pm 0.004$ \\
亚硝态氮 $\left(\mathrm{NO}_{2}^{-}-\mathrm{N}\right)$ & $0.037 \pm 0.001$ \\
溶解性有机氮 $(\mathrm{DON})$ & $0.339 \pm 0.071$ \\
尿素氮 $(\mathrm{Urea}-\mathrm{N})$ & $0.175 \pm 0.063$ \\
总磷 $(\mathrm{TP})$ & $0.036 \pm 0.021$ \\
溶解性无机磷 $(\mathrm{DIP})$ & $0.012 \pm 0.007$ \\
溶解性有机磷 $(\mathrm{DOP})$ & $0.024 \pm 0.015$ \\
\hline
\end{tabular}

于 2010 年 6 月太湖蓝藻水华暴发期间, 用 $25^{\#}$ 浮游生物 网从梅梁湾捞取藻浆 (生物量 $98 \%$ 为微囊藻). 将藻浆于水 桶中静置分层, 选取漂浮在上层的藻浆, 用蒸馏水洗涤, 分层 和洗涤 3 次, 置于烘箱中, $60{ }^{\circ} \mathrm{C}$ 烘干, 研磨过篮后待用. 实验 用蓝藻碎屑的总氮含量为 $88.56 \pm 4.10 \mathrm{mg} / \mathrm{g}$, 总磷含量为 $6.88 \pm 0.61 \mathrm{mg} / \mathrm{g}$, 蓝藻碎屑的起始烧失重与氮含量比 $(\mathrm{AFDW} / \mathrm{TN})$ 为 $10.40 \pm 0.08$.

实验用水取自太湖梅梁湾, 静置后用 GF/C 膜过滤备 用,其初始水化指标见表 1 .

\section{2 实验设计}

实验于 $500 \mathrm{ml}$ 雉型瓶中进行, 每个雉型瓶中加人 $\mathrm{GF} / \mathrm{C}$ 膜过滤后的湖水 $250 \mathrm{ml}$, 共设置 18 个雉型瓶, 置于实验室通 风透光处, 实验期间水温保持在 $25-30^{\circ} \mathrm{C}$ 之间.

实验共进行 10 天, 实验开始前一天测定实验用水的初 始指标, 并在早晨 8 点向每个实验装置中加人烘干后的蓝藻

碎屑 $0.100 \mathrm{~g}(250 \mathrm{ml}$ 叶绿素浓度为 $1600 \mu \mathrm{g} / \mathrm{L}$ 的水体所含蓝藻烘干后形成的碎屑 $)$, 其中包含 $8.856 \pm$ $0.410 \mathrm{mg}$ 的氮以及 $0.688 \pm 0.061 \mathrm{mg}$ 的磷. 分别在加人蓝藻碎屑后第 $1 、 2 、 3 、 4 、 7 、 10 \mathrm{~d}$, 随机取三个雉型瓶作 为重复, 测定相关指标.

\section{3 测定指标及方法}

实验开始前预留部分培养用水以及实验用蓝藻碎屑, 分别用于测定培养用水初始水化指标, 实验用蓝 藻碎屑氮、磷含量以及烧失重 (AFDW). 取样时间定在上午 $8: 00$, 每次取样将雉形瓶中水样混匀后取 $50 \mathrm{ml}$, 用 $550{ }^{\circ} \mathrm{C}$ 灼烧过的 $\mathrm{GF} / \mathrm{C}$ 滤膜过滤, 滤膜用于测定悬浮质及悬浮质烧失重, 过滤后水样用于测定水化指标.

水化指标包括水样中 $\mathrm{TN} 、 \mathrm{NH}_{4}^{+}-\mathrm{N} 、 \mathrm{NO}_{3}^{-}-\mathrm{N} 、 \mathrm{NO}_{2}^{-}-\mathrm{N} 、$ Urea- $\mathrm{N} 、 \mathrm{TP} 、$ 溶解性反应磷 ( $\mathrm{SRP}$ ), 并计算水样中 TDN 、DIN 、DON、TDP、DIP 和 DOP 的含量.

$\mathrm{TN}$ 、TP 含量采用碱性过硫酸钾联合消解法测定; $\mathrm{NH}_{4}^{+}-\mathrm{N}$ 含量采用纳氏试剂比色法测定; $\mathrm{NO}_{3}^{-}-\mathrm{N}$ 含量采 用紫外分光光度法测定; $\mathrm{NO}_{2}^{-}-\mathrm{N}$ 含量采用 $\mathrm{N}-\left(1\right.$ - 䒬基) -乙二胺光度法测 ${ }^{\text {定 }}{ }^{[10]}$; Urea- $\mathrm{N}$ 含量采用二乙酰一肜 比色法测定 ${ }^{[11]}$; SRP 含量采用钿-锑-抗分光光度法测定 ${ }^{[10]}$. AFDW 为悬浮质于 $550{ }^{\circ} \mathrm{C}$ 下灼烧 $4 \mathrm{~h}$ 后的损失质 量; $\mathrm{AFDW} / \mathrm{TN}$ 为蓝藻碎屑 $\mathrm{AFDW}$ 与氮含量的比值 $(\mathrm{AFDW} / \mathrm{TN} \text { 可反映物质的 } \mathrm{C} / \mathrm{N})^{[12]}$.

\section{4 分解速率计算方法}

分解速率采用 Olsen 的指数衰减模型计算: $w_{t} / w_{0}=\mathrm{e}^{-k t}$, 式中, $w_{0}$ 为蓝藻碎屑初始干重, $w_{t}$ 为分解 $t$ 时间 后剩余蓝藻碎屑干重, $t$ 为分解时间, $k$ 即为分解速率 ${ }^{[13]}$. 
氮、磷释放速率为添加蓝藻碎屑中所含氮、磷损失百分比与时间的比值, 公式为 $\left(1-W_{t} / W_{0}\right) / t$, 其中 $W_{0}$ 为水体中初始蓝藻碎屑氮、磷含量, $W_{t}$ 为分解 $t$ 时间后水体中剩余蓝藻碎屑氮、磷含量, $t$ 为分解时间.

\section{5 数据处理}

实验结果均用平均值 \pm 标准差 $(\mathrm{X} \pm \mathrm{SD})$ 表示, 使用 SPSS 16.0 软件在 $\alpha=0.05$ 水平下以时间为因素进 行方差分析,部分数据采用 Duncan 法进行组间差异的多重比较.

\section{2 结果}

\section{1 蓝藻碎屑分解速率}

实验期间,蓝藻碎屑剩余物质干重随时间变化显著 $(P<0.05)$, 在开始阶段剩余物质干重迅速减小, 至实 验第 $2 \mathrm{~d}$ 蓝藻碎屑平均分解了 $41.9 \%$, 蓝藻碎屑的分解速率在第 $2 \mathrm{~d}$ 也达到最高, 显著高于其它时间 $(P<0.05)$; 第 3-7 d, 蓝藻碎屑剩余物质干重逐渐趋于稳定, 蓝藻碎屑分解速率显著降低 $(P<0.05)$; 之后蓝 藻碎屑分解速率也趋于稳定, 到第 $10 \mathrm{~d}$ 时, 蓝藻碎屑分解速率只略小于第 $7 \mathrm{~d}$, 差异不再显著 $(P>0.05)$ (图 1).

\section{2 蓝藻碎屑分解过程中碎屑氮、磷释放速率及水体 TDN 和 TDP 浓度的变化}

在蓝藻碎屑分解的过程中,氮和磷的释放并不是同速的 (图 2), 在分解的前 $4 \mathrm{~d}$,磷的释放速率远大于氮 的释放速率, 之后两者趋于相同, 其中氮的释放速率与蓝藻碎屑分解速率变化趋势相似, 可见氮的释放随干 物质的分解进行.

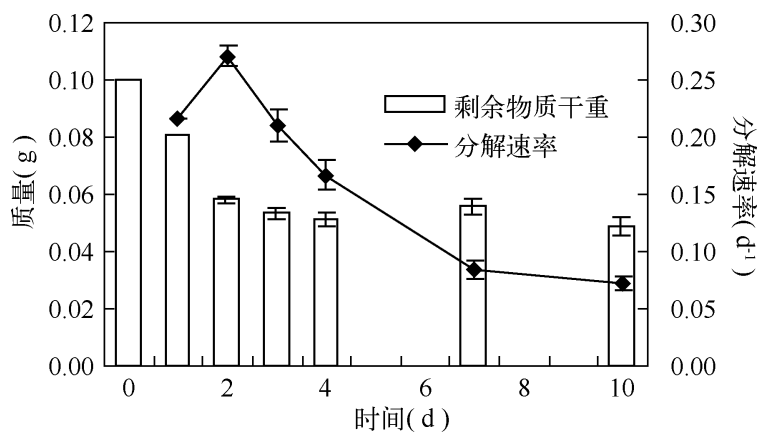

图 1 蓝藻碎屑分解过程中剩余物质干重及 蓝藻碎屑分解速率

Fig. 1 Remaining dry mass and the decomposition rate of detritus during the decomposing of cyanobacteria

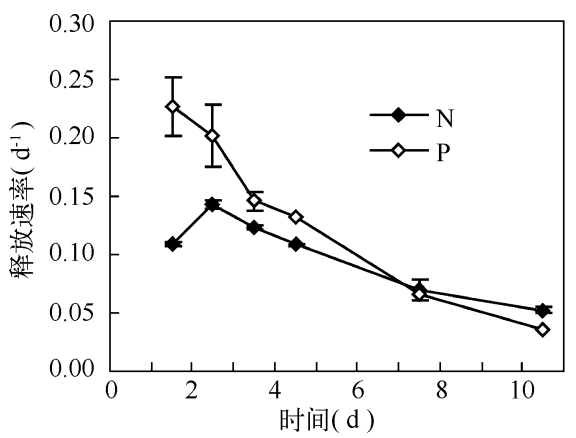

图 2 蓝藻碎屑分解过程中氮、磷释放速率

Fig. 2 Release rates of nitrogen and phosphorus from the decomposing cyanobacterial detritus

在整个实验过程中 TDN、TDP 浓度均随时间变化显著 $(P<0.05)$, 其中 TDN 浓度不断增加 ( 图 3a), 特
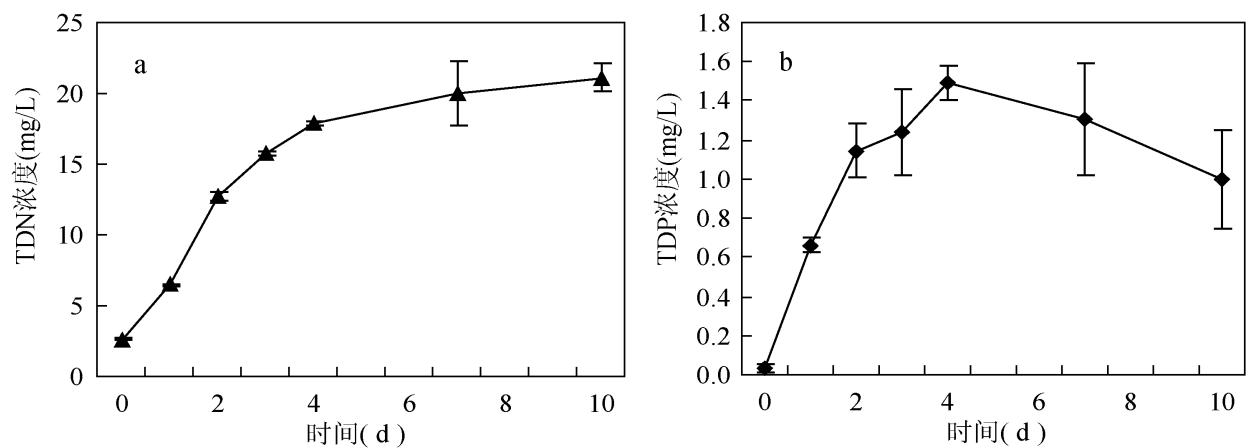

图 3 蓝藻碎屑分解过程中水体 $\operatorname{TDN}(\mathrm{a})$ 和 TDP $(\mathrm{b})$ 浓度变化

Fig. 3 Concentrations of $\operatorname{TDN}(\mathrm{a})$ and $\operatorname{TDP}(\mathrm{b})$ during the decomposing of cyanobacteria detritus 
别是实验的前 $4 \mathrm{~d}$, 水中 TDN 浓度每天均相对前一天显著增加 $(P<0.05) .10 \mathrm{~d}$ 内, 水体中 TDN 增加量相当 于所添加蓝藻氮含量的 $52.3 \%$; 蓝藻碎屑在加人到水中后, 磷释放很快, 第 $1 \mathrm{~d}$ 水体中 TDP 浓度就显著增加 $(P<0.05)$, 在分解 $4 \mathrm{~d}$ 后 TDP 浓度达到最大, 之后变化不再显著 $(P>0.05)$ (图 $3 \mathrm{~b}$ ).

\section{3 蓝藻碎屑分解过程中水体 DIN 和 DON 含量的变化}

蓝藻碎屑在分解过程中, 不论是 DIN 浓度还是 DON 浓度都呈现出增加的趋势, 并且随时间的变化差异 显著 $(P<0.05$, 图 4a) ; 但两者又略有不同, 其中 DIN 的浓度在分解的前 $4 \mathrm{~d}$ 快速增加, 之后则趋于稳定; 而 DON 浓度的增加则从分解之初开始, 一直持续到实验结束.

蓝藻碎屑分解过程中 DIN 各组分浓度均随时间变化显著 $(P<0.05$ ), 但趋势并不相同 (图 4b), 其中 $\mathrm{NH}_{4}^{+}-\mathrm{N}$ 浓度在加藻后显著升高 $(P<0.05)$, 并在第 $4 \mathrm{~d}$ 达到最大值; 蓝藻分解后, $\mathrm{NH}_{4}^{+}-\mathrm{N}$ 在 DIN 中所占的比 例很高, 加藻后第 $2 \mathrm{~d}$ 就达到 $87.9 \%$, 以后则维持在 $94.0 \%$ 左右, 而在初始水样中, $\mathrm{NH}_{4}^{+}-\mathrm{N}$ 所占的比例仅为 $10.2 \%$ 左右. 而 $\mathrm{NO}_{3}^{-}-\mathrm{N}$ 和 $\mathrm{NO}_{2}^{-}-\mathrm{N}$ 浓度在蓝藻分解后相比初始值都显著下降 $(P<0.05)$.

在 DON 中, Urea-N 在加藻后, 略有增加但其随时间的变化并不显著 $(P>0.05)$, 且在 DON 中所占的比 例显著减少 $(P<0.05)$, 由加藻前的 $56.0 \%$, 到加藻后第 $2 \mathrm{~d}$ 开始降到平均 $9.4 \%$, 至第 $10 \mathrm{~d}$ 实验结束时, Urea-N在 DON 中所占的比例仅为 3.0\% (图 4c), 说明蓝藻碎屑分解释放的有机氮中, 尿素并不是主要形式.

\section{4 蓝藻碎屑分解过程中水体 DIP 和 DOP 含量的变化}

水体中 DIP、DOP 浓度随时间的变化差异显著 $(P<0.05)$, 其中 DIP 浓度在蓝藻加人后 $7 \mathrm{~d}$ 内呈现上升 趋势 (图 5$)$, 此后 DIP 浓度变化差异不再显著 $(P>0.05)$, 蓝藻碎屑分解过程中 DIP 所占的比例也呈现出上 升的趋势, 而 DOP 的浓度则在蓝藻加人后前 $4 \mathrm{~d}$ 呈现上升趋势, 至第 $7 \mathrm{~d}$ 时, 相对于第 $4 \mathrm{~d}$ 显著减小 $(P<$ $0.05)$, 之后变化不再显著 $(P>0.05)$.

\section{3 讨论}

\section{1 蓝藻碎屑分解速率}

浮游藻类和水生高等植物是水生态系统中的两种重要初级生产者, 浮游藻类利用水中的营养盐, 水生 高等植物则既可以利用水中的营养盐也可以利用沉积物中的营养盐 ${ }^{[14]}$, 同时两者都会在死亡调落后通过分 解向水体中释放营养盐, 从而参与水生态系统的营养盐循环. 相比于水生高等植物, 蓝藻碎屑的分解速度更 快, 在本实验中, 其在短短的 $4 \mathrm{~d}$ 内就可以分解物质干重的 50\% 左右, 而水生高等植物则通常需要 $0.5-12$ 个月 ${ }^{[15]}$. 通过计算发现蓝藻碎屑在水体中的分解速率在 $0.072-0.271 \mathrm{~d}^{-1}$ 之间, 远大于一般水生高等植物 的分解速率 ${ }^{[16-17]}$, 因此可以推断出藻类在营养物质循环中的周转速率比水生植物更快. 造成这种现象的原 因与蓝藻碎屑物理化学性质有关:一方面蓝藻碎屑相比于植物腐烂所形成的残体, 颗粒更小, 表面积与体积 比更大, 从而增大了分解材料与水体的接触和交换; 另一方面则与蓝藻碎屑的化学组成有关, 有学者发现 N 含量较高的有机物, 分解更快 ${ }^{[18-19]}$, 这是由于碎屑颗粒的分解主要依靠环境中微生物的降解作用, 而微生物 生长对环境中的 $\mathrm{C} 、 \mathrm{~N} 、 \mathrm{P}$ 含量及比例有一定的要求, 当微生物生长基质中 $\mathrm{C}: \mathrm{N}: \mathrm{P}$ 达到 106:12:1 时, 最有利 于微生物的生长 ${ }^{[20]}$. 由于大型植物需要更多的碳用于形成茎、叶、根等支撑系统, 故其往往有更高的碳含量, 有学者发现浮游植物 $C 、 N 、 P$ 原子比为 110: 16: 1, 而大型水生植物原子比为 500: 24: $1^{[21-22]}$, 本实验中测得蓝 藻碎屑 $\mathrm{AFDW} / \mathrm{TN}$ 平均为 10.40 , 而水生植物残体多在 34 以上, 也可看出蓝藻碎屑有着更高的氮含量, 且蓝 藻碎屑 $\mathrm{N} / \mathrm{P}$ 平均为 12.87 , 其 $\mathrm{C}: \mathrm{N}: \mathrm{P}$ 相对于水生植物残体更符合微生物的生长需求, 因此蓝藻碎屑相比于 水生植物有着更高的分解速率及分解率. 较高的分解率使蓝藻碎屑相对于水生植物残体而言, 短期内可能 会有更大比例的营养物质以溶解态营养盐的形式留在上覆水或间隙水中, 而不是以颗粒物形式进人沉积物 中, 从而很快被水体中的初级生产者利用.

\section{2 蓝藻碎屑分解过程中的营养盐释放}

蓝藻碎屑在分解的过程中将释放大量氮、磷营养盐, 在 $10 \mathrm{~d}$ 之内, 均有超过蓝藻碎屑本身含量 $50 \%$ 的 氮、磷被分解释放到水体中, 其中磷的释放速率在实验前 $4 \mathrm{~d}$ 要高于氮, 这可能是由于磷主要存在于利于分 解的活性物质中造成的 ${ }^{[15]}$.

蓝藻碎屑分解过程中氮主要通过被细菌或浮游动物矿化形成 $\mathrm{NH}_{4}^{+}-\mathrm{N}$ 以及自溶产生 $\mathrm{DON}$ 两条途径释 
放 ${ }^{[23]}$, 所以实验前 $4 \mathrm{~d}$ DIN 、DON 浓度均显著上 升,且 DIN 中 $\mathrm{NH}_{4}^{+}-\mathrm{N}$ 的比例超过 $90 \%, 4 \mathrm{~d}$ 之后 DIN 浓度趋于稳定, 而 DON 浓度仍在升高, 这 表明蓝藻碎屑分解后期,DON 释放大于 DIN. 蓝 藻碎屑产生的 DON 中, Urea-N 浓度虽略有增 加,但 Urea-N 占 DON 的百分比始终较低, 说明 蓝藻碎屑释放的主要为非尿素形式的有机态 氮,有可能是氨基酸、肽段或蛋白质等形式. 对 于磷营养盐而言, 初始水体中 DIP、DOP 浓度均 很低,因而蓝藻碎屑的快速分解使得两者浓度 在实验前 $4 \mathrm{~d}$ 均显著增加, 而此后蓝藻碎屑中磷 的释放速度减缓, 水中 TDP 浓度趋于稳定, 而 DOP 逐步转化为 DIP, 故第 4-7d, DOP 浓度降 低而 DIP 浓度增加, 第 $7 \mathrm{~d}$ 之后, DIP 及 DOP 均 趋于稳定.

\section{3 蓝藻营养盐释放可能导致的生态后果}

蓝藻分解过程中释放了大量的无机营养 盐, 其中 $90 \%$ 都是 $\mathrm{NH}_{4}^{+}-\mathrm{N}$, 作为生物最容易利 用的氮源, 它将很快被水生生态系统中藻类、微 生物等利用,成为其重要的营养来源. 同样, 本研 究也发现蓝藻碎屑分解释放了一定比例的有机态 氮磷产物, 特别是 DON, 蓝藻分解使水中的 DON 浓度及 DON 占 TDN 百分比大幅上升, 而已有研 究表明不论是有机态氮还是有机态磷, 都可以 被水生生态系统中的生物, 包括微生物、浮游植 物和水生植物直接利用 ${ }^{[24-26]}$, 特别是水华常见 藻类如: 束丝藻 (Aphanizomenon flosaquate)、铜 绿微囊藻 (Microcystis aeruginosa) 相比于其他藻 类对有机氮的吸收能力更强, 在有机氮浓度较 高的环境中能更好地生长 ${ }^{[27-28]}$, 这种对有机营 养盐的直接吸收利用使得蓝藻碎屑在湖泊营养 盐循环过程中周转更加迅速.

局部湖区发生的蓝藻水华,有可能会在风 及水流的作用下发生长距离的漂移 ${ }^{[8]}$, 将大量 有机碎屑输送到湖滨带, 且蓝藻水华漂浮物容 易被湖滨带的水生植物区捕获, 并在此腐烂分 解, 而蓝藻碎屑的快速分解就使其迅速成为可 被岸带大型水生植物利用的营养盐来源 ${ }^{[29-30]}$, 从而对整个湖泊生态系统产生重要的影响. 一
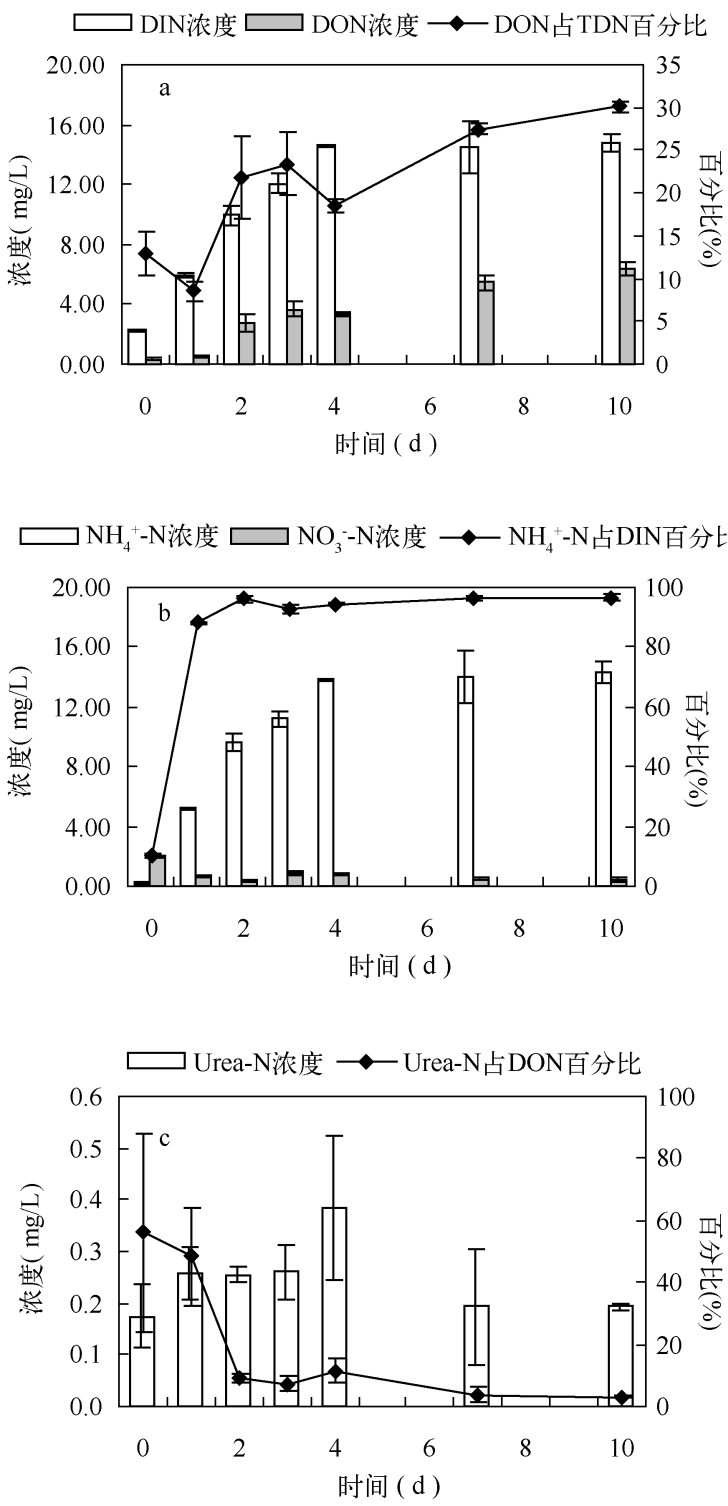

图 4 蓝藻碎屑分解过程中水体不同形态氮 ( a:DON、DIN; $\mathrm{b}: \mathrm{NH}_{4}^{+}-\mathrm{N} 、 \mathrm{NO}_{3}^{-}-\mathrm{N} ; \mathrm{c}:$ Urea-N) 的浓度及相应比例

Fig. 4 Concentrations and percentages of different forms of nitrogen ( a: DON, DIN; b: ammonia, nitrate;

c: Urea-N) in the water column during the decomposing of cyanobacteria detritus 方面, 岸带大型水生植物获得了更多的营养盐, 这也可能是岸带, 尤其是下风区岸带大型水生植物生产力的 重要物质基础; 另一方面,岸带大型水生植物对这部分来自敞水区营养盐的吸收,会增加营养盐的滞留,否 则这部分营养盐会重新被浮游植物吸收利用,而成为维持高密度浮游植物或水华的重要营养盐来源 ${ }^{[31]}$. 当 然, 过多的蓝藻堆积, 则有可能会影响大型水生植物, 尤其是沉水植物的生长, 甚至导致其死亡, 这种影响显 然与蓝藻堆积的程度与时间有关系, 而这种关系还有待进一步研究. 


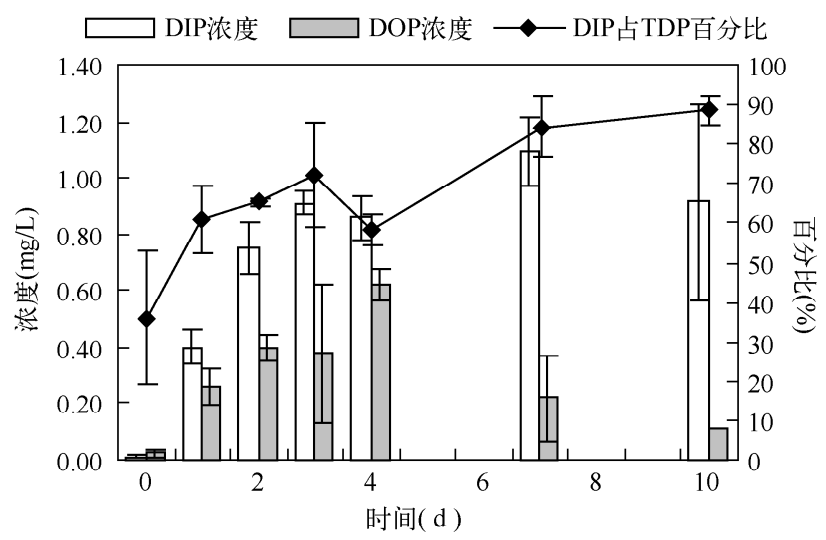

图 5 蓝藻碎屑分解过程中水体 DIP 和 DOP 浓度及 TDP 中 DIP、DOP 所占百分比

Fig. 5 Concentrations and percentages of DIP and DOP in the water column during decomposing of cyanobacteria detritus

\section{4 结语}

蓝藻碎屑在湖水中能够很快分解并释放出大量氮、磷营养盐; 其中磷的释放速率要高于氮; 在实验期间 蓝藻分解产生的 TDN 中既包括 DIN 也包括一定比例的 DON, 且 DON 在 TDN 中的比例逐渐增加; 而蓝藻碎 屑分解产生的 TDP 中, 初期会有一定比例的 DOP, 但第 $4 \mathrm{~d}$ 以后, DOP 会逐渐减少, 转化为 DIP, 并逐渐趋于 稳定; 由于蓝藻碎屑的这些分解特性, 其迁移及分解的过程将不可避免地对湖泊中的营养盐循环产生影响; 本实验中通过对蓝藻碎屑分解释放营养盐速率及形态的探寻, 为研究水生植物对不同形态氮磷营养盐利用 的后续研究打下一定基础, 同时也将有助于今后从生态系统的角度继续研究了解水华发生对湖泊营养盐循 环产生的影响.

\section{5 参考文献}

[1 ] 全为民, 沈新强, 严力蛟. 富营养化水体生物净化效应的研究进展. 应用生态学报, 2003, 14(11) : 2057-2061.

[ 2 ] 孔繁翔, 马荣华, 高俊峰等. 太湖蓝藻水华的预防、预测和预警的理论与实践. 湖泊科学, 2009, 21 (3): 314-328.

[ 3 ] 李敦海, 李根保, 王高鸿等. 水华蓝藻生物质对沉水植物五刺金鱼藻生长的影响. 水生生物学报, 2007, 31 (5): 689-692.

[ 4 ] 孙小静, 秦伯强, 朱广伟. 蓝藻死亡分解过程中胶体态磷、氮、有机碳的释放. 中国环境科学, 2007, 27(3): 341345 .

[ 5 ] Krivtsov V, Bellinger EG, Sigee DC. Elemental composition of Microcystis aeruginosa under conditions of lake nutrient depletion. Aquatic Ecology, 2005, 39: 123-134.

[ 6 ] 姜 霞, 钟立香, 王书航等. 巢湖水华暴发期水一沉积物界面溶解性氮形态的变化. 中国环境科学, 2009, 19 (11) : 1158-1163.

[ 7 ] Nakamura Y, Watanabe MM. Growth characteristics of Chattonella antique. Part 2. Effects of Nutrients on Growth. Journal of the Oceanographical Society of Japan, 1983, 39: 151-155.

[8] 朱广伟. 太湖富营养化现状及原因分析. 湖泊科学, 2008, 20(1) :21-26.

[ 9 ] 王洪君, 王为东, 卢金伟等. 植被型岸边带对藻类的捕获与水源保护研究. 中国给水排水, 2006, 22(7): 1-3.

[10］金相灿，屠清瑛. 湖泊富营养化调查规范. 北京: 中国环境科学出版社, 1990.

[11］严小蓉. 游泳池水中尿素检测方法改进. 中国实用医药, 2009, 4(5): 19-27.

[12] 厉恩华, 刘贵华, 李 伟等. 洪湖三种水生植物的分解速率及氮、磷动态. 中国环境科学, 2006, 26 (6) : $667-671$. 
[13 ] Olson JS. Energy storage and the balance of products and decomposers in ecological system. Ecology, 1963, 44(2) : 322331.

[14] Lee K, Dunton KH. Inorganic nitrogen acquisition in the seagrass Thalassia testudium: Development of a whole-plant nitrogen budget. Limnology and Oceanography, 1999, 44(5) : 1204-1215.

[15］李文朝, 陈开宁, 吴庆龙等. 东太湖水生植物生物质腐烂分解实验. 湖泊科学, 2001, 13(4): 331-336.

[16] Battle JM, Mihuc TB. Decomposition dynamics of aquatic macrophytes in the lower Atchafalaya, a large flood plain river. Hydrobiologia, 2000, 418: 123-136.

[17] 潘慧云, 徐小花, 高士祥. 沉水植物衰亡过程中营养盐的释放过程及规律. 环境科学研究, 2008, 21 (1) : 64-68.

[18 ] Menéndez M, Martinez M, Hernández $\mathrm{O}$ et al. Comparison of leaf decomposition in two mediterranean rivers: a large eutrophic river and an oligotrophic stream( S Catalonia, NE Spain). International Review of Hydrobiology, 2001, 86(4-5) : 475-486.

[19] Anderson JT, Smith LM. The effects of flooding regimes on decomposition of Polygonum pensylvanicum in the playa wetlands( Southern Great Plains, USA). Aquatic Botany, 2002, 74: 97-108.

[20] Enriquez S, Duarte CM, Sand-Jensen K. Patterns in decomposition rates among photosynthetic organisms: the importance of detritus C: N: P content. Oecologia, 1993, 94 : 457-471.

[21] Cebrian J, Duarte CM. The dependence of herbivory on growth rate in natural plant communities. Functional Ecology, 1994, 8 : 518-525.

[22] Sand-Jensen K, Borum J. Interactions among phytopalnkton, periphyton, and macrophytes in temperate freshwaters and estuaries. Aquatic Botany, 1991, 41(1-3): 137-175.

[23] 曾 巾, 杨柳燕, 肖 琳等. 湖泊氮素生物地球化学循环及微生物作用. 湖泊科学, 2007, 19(4): 382-389.

[24] Vonk JA, Middelburg JJ, Stapel J et al. Dissolved organic nitrogen uptake by seagrasses. Limnology and Oceanography, $2008, \mathbf{5 3}(2): 542-548$.

[25] Glibert PM, Heil CA, Revilla M et al. Evidence for dissolved organic nitrogen and phosphorus uptake during a cyanobacterial bloom in Florida Bay. Marine Ecology Progress Series, 2004, 280 : 73-83.

[26] 沈 宏, 宋立荣, 周培疆等. 有机磷农药对滇池微囊藻生长和摄磷效应的影响. 水生生物学报, 2007, 31 (6): 863-868.

[27] Berman T. Dissolved organic nitrogen utilization by an Aphanizomenon bloom in Lake Kinneret. Journal of Plankton Research, 1997, 19(5) : 557-586.

[28] Berman T, Chava S. Algal growth on organic compounds as nitrogen sources. Journal of Plankton Research, 1999,21 (8) : 1423-1437.

[29] Li KY, Liu ZW , Gu BH. The fate of cyanobacterial blooms in vegetated and unvegetated sediments of a shallow eutrophic lake: A stable isotope tracer study. Water Research, 2010, 44: 1591-1597.

[30] Zhang LY, Li KY, Liu ZW et al. Sedimented cyanobacterial detritus as a source of nutrient for submerged macrophytes (Vallisneria spiralis and Elodea nuttallii): An isotope labeling experiment using ${ }^{15} \mathrm{~N}$. Limnology and Oceanography, 2010, 55 (5) : 1912-1917.

[31 ] Barron C, Middelburg JJ, Duarte CM. Phytoplankton trapped within seagrass(Posidonia oceanica) sediments are a nitrogen source: An in situ isotope labeling experiment. Limnology and Oceanography, 2006, 51(4) : 1648-1653. 